\title{
Tumour Heterogeneity of Breast Cancer: From Morphology to Personalised Medicine
}

\author{
Mohammed A. Aleskandarany ${ }^{a}$ Michel E. Vandenberghe ${ }^{b}$ Caterina Marchiò ${ }^{c}$ \\ Ian O. Ellis ${ }^{a}$ Anna Sapino ${ }^{c, d}$ Emad A. Rakha ${ }^{a}$ \\ aDepartment of Histopathology, University of Nottingham and Nottingham University Hospitals NHS Trust, City \\ Hospital, Nottingham, and b Precision Medicine Laboratories, Precision Medicine and Genomics, IMED Biotech Unit, \\ AstraZeneca, Cambridge, UK; ${ }^{C}$ Department of Medical Sciences, University of Turin, Turin, and d Candiolo Cancer \\ Institute-FPO, IRCCS, Candiolo, Italy
}

\section{Keywords}

Breast cancer · Heterogeneity · Morphology ·

Molecular heterogeneity · Microenvironment · Sampling ·

Personalised therapy · Resistance

\begin{abstract}
Breast cancer (BC) displays striking clinical, morphological, and behavioural diversity within a single tumour and between tumours. Currently, mounting evidence indicates that the morphological heterogeneity of $\mathrm{BC}$ reflects an underlying spectrum of genetic and epigenetic portraits that control $B C$ behaviour. Further understanding of $B C$ heterogeneity will have an impact, not only on the routine diagnostic practices but also on patients' management decisions. Phenomena like diagnostic inconsistencies and therapeutic resistance, both primary and acquired, could be attributed, at least in part, to tumour heterogeneity within the same cancer and between the primary disease and subsequent recurrences. From a practical standpoint, and to minimise the impact of BC intratumoral heterogeneity, pragmatic approaches for adequate tumour sampling have been suggested in translational biomarker discovery and validation research
\end{abstract}

\section{KARGER}

(c) 2018 S. Karger AG, Basel

E-Mail karger@karger.com

www.karger.com/pat studies and in the clinical setting. Here, we provide a brief overview of $\mathrm{BC}$ heterogeneity, with an emphasis on the clinical consequences of intratumoral heterogeneity.

() 2018 S. Karger AG, Basel

\section{Introduction}

Breast cancer $(\mathrm{BC})$ is characterised by a remarkable degree of morphological and molecular heterogeneity, not only between tumours (intertumoral heterogeneity) but also among the same tumours (intratumoral heterogeneity). At the level of an individual patient's tumours, heterogeneity can also be classified into "spatial" and "temporal." Temporal heterogeneity is particularly important when comparing the features of the primary tumour and metastatic or recurrent lesions, and pre-invasive and invasive disease in the same tumours. There is sufficient evidence to indicate considerable differences between the primary tumour and local or distant recurrences that may have an impact on the treatment decisions for patients with recurrent disease [1-3]. Heterogeneity in $\mathrm{BC}$ is the result of a co-ordinated interplay be- 
tween the tumour cells themselves and also between the tumour cells and the different stromal elements. Understanding this complex interplay is a fundamental requisite to address how the tumour progresses and why some therapeutic regimens are initially or subsequently ineffective. In this review, we discuss BC heterogeneity within the context of tumour evolution and progression, taking into account the impact on disease diagnosis and research studies for biomarker identification. In parallel, the importance of the tumour microenvironment (TME) and the contribution of rapidly developing next-generation sequencing (NGS) in deciphering the origin and consequences of heterogeneity are discussed. The impact of molecular heterogeneity on the response to the development of targeted therapy as well as efficacy and resistance will also be addressed.

\section{Heterogeneity: Notions from Morphology}

Although genetic and molecular heterogeneity in BC has largely been dissected in studies based on single-cell analysis, morphology is often neglected. Morphological features including histological type and grade vary among BC patients, with three grades and $>20$ histological types having been described; these are known to impact on $\mathrm{BC}$ patient outcome $[4,5]$.

Tubule formation, cytonuclear pleomorphism, and mitotic counts, the 3 components of histological grade, often vary in different areas of the tumour. Grading is based on the overall percentage of tubule formation combined with the highest mitotic count and highest degree of cytonuclear pleomorphism [6]. The heterogeneous "mixed" histotypes constitute up to $20 \%$ of cases, featuring some characteristic special-type areas together with areas showing no special features. In the subtyping of $\mathrm{BC}$, the presence of a minor component of a specific tumour type in $<10 \%$ of the tumour does not change tumour typing, and the tumour remains in the "pure" category. Some histological types such as tubular or invasive cribriform carcinomas are associated with an excellent prognosis, and can be treated by endocrine therapy alone when present as pure forms $[7,8]$; however, if these tumours contain a component of another histotype, their excellent prognosis may change and the therapeutic approach should then be remodelled according to the worse biological features. Metaplastic breast carcinoma (MBC), a rare entity of $\mathrm{BC}(1-2 \%)$, is an obvious illustration of intratumoral morphological phenotypic heterogeneity. $\mathrm{MBC}$ can feature squamous epithelium or mesenchymal components in pure or mixed forms and in variable proportions, with or without conventional adenocarcinomatous elements [9]. In their proof-of-principle study, Geyer et al. [10], who micro-dissected phenotypically distinct tumour zones from six MBCs, demonstrated diversity between the genetic profiles and morphology of the different components within the same tumours. Morphologically distinct components from each case were clonal and harboured remarkably similar genetic profiles; however, in two cases, morphologically distinct components harboured additional genetic aberrations specific to certain tumour types. Therefore, the intratumoral phenotypic diversity could have resulted from distinct genetic aberrations in the morphologically distinct clones, or resulted from other mechanisms such as epigenetic events or alterations in certain pathways in genetically similar areas.

Mammary glandular cells show a high degree of phenotypic plasticity, which can be appreciated in both benign and malignant conditions [11-13]. These phenotypic alterations represent the expression of genotypic and molecular properties not present in normal mammary glandular cells, and in benign as well as some malignant conditions they are likely to be the result of de-repression of the normally coded molecular mechanisms responsible for the epithelial phenotype rather than histogenesis [11-13]. In support of this, some authors have demonstrated that basal-like BC may arise from luminal progenitor cells [14]. Metaplastic trans-differentiation has been demonstrated, not only in the in-situ and invasive lesions but also at distant metastatic sites [12]. The phenomenon of epithelial-mesenchymal transition is welldocumented in $\mathrm{BC}$ where malignant epithelial cells lose their junctional structures, express mesenchymal proteins, and remodel their extracellular matrix (ECM). These phenotypic alterations can be observed in the whole tumour, but, not infrequently, they are present focally and therefore contribute to intratumoral heterogeneity. Finally, other morphological features of $\mathrm{BC}$ also differ, including the presence and extent of associated duct carcinoma in situ (DCIS), lymphovascular invasion, the degree of tumour-infiltrating inflammatory cells, and the nature of tumour-associated stroma.

\section{Heterogeneity in the Sequencing Era: Proposed Origin}

The analysis of $\mathrm{BC}$ samples and single-cell analysis by NGS, as identified by whole-genome and exome sequencing, have advanced our understanding of the disease and 
portrayal of the genetic and epigenetic landscapes [15]. NGS has provided further evidence to suggest that intratumoral genetic heterogeneity likely plays a fundamental role in the phenotypic heterogeneity observed in BC [15]. Two theories have been currently adopted to explain tumour heterogeneity, namely the cancer stem-cell (CSC) hypothesis [16] and the clonal evolution and selection model [17]. It is widely accepted that these concepts are complementary rather than being mutually exclusive [18]. The tumour microenvironment also poses internal selective pressure which could fuel cellular heterogeneity. In the CSC hypothesis model, only a small fraction of cells can initiate and drive tumour progression, while in the clonal evolution model, initiation and progression follow a Darwinian-like evolutionary pattern where cellular clones that would be able to progress emerge over time. Both models suggest the existence of cells with different genetic profiles within the tumour, aside from the difference in the mechanisms that are assumed to be at work. In the CSC model, some tumour features such as therapy resistance could be an inherent trait, but in the clonalevolution model, features of aggressiveness including resistance to therapy are considered as an emerging timedependent cumulative attribute $[19,20]$.

\section{BC Heterogeneity at the Molecular Level}

Different authorities have studied the heterogeneity of $\mathrm{BC}$ at the molecular level. For instance, Park et al. [21], evaluating the genomic imbalance assessed by fluorescence in situ hybridisation (FISH) in different cell immunotypes in both in situ and invasive tumours, demonstrated a high degree of genetic heterogeneity within and between distinct tumour cell populations, based on cellular phenotype markers, including stem-cell-like characteristics. The degree of diversity correlated with clinically relevant BC subtypes. Using sector-ploidy profiling of isolated nuclei obtained from different areas of the tumour, Navin et al. [2] demonstrated the existence of monogenomic and polygenomic breast cancers. They also studied clonality on the basis of copy-number alterations by single-cell sequencing of tumour sectors. Results showed that monogenomic tumours may have a single clonal expansion in primary and liver metastasis whereas polygenomic tumours may show up to three distinct clonal subpopulations, probably representing sequential clonal expansions. In addition, they defined an abundant subpopulation of genetically diverse "pseudodiploid" cells that do not travel to the metastatic site.

Breast Cancer Heterogeneity

\section{Molecular Heterogeneity at the Gene Expression Level}

In the last decade, with the advances in $\mathrm{BC}$ profiling using high-throughput gene expression microarrays, multiple molecular subtypes of $\mathrm{BC}$ have been identified [22-24]. These subtypes, defined by their distinct transcriptional signatures, resulted in a dramatic paradigm shift in our understanding of BC heterogeneity [25]. The different subtypes identified showed prognostic value independent of tumour morphological features and also some tumours with variable morphology clustered together in certain molecular classes or intrinsic subtypes [26]. They also showed a significant degree of genetic heterogeneity, with different patterns of inter- and intrachromosomal rearrangements and DNA mutational landscapes. Using different approaches such as integrated gene-expression profiling and DNA copy number [27], or tissue microarrays (TMA) and large panels of immunohistochemical markers [28-30], similar molecular subtypes were identified with distinct clinical outcomes as well as response to therapies. However, heterogeneity still exists within the most of these defined classes. The luminal type, principally enriched for oestrogen receptor (ER) and ER-regulated genes, could be subdivided into at least two subclasses, luminal $A$ and $B$, with a difference in the level of expression of certain genes including ER-related genes, proliferation genes, and HER2 [31]. The basal-like subtype generally, but not perfectly, corresponds to the triple-negative $\mathrm{BC}$ (negative for ER, progesterone receptor [PR], and HER2) [43, 33]. This subtype features substantial heterogeneity where some specific molecular subtypes have been characterised, including the claudinlow subtype (with poor outcome) and the molecular apocrine subtype (exhibiting positivity for androgen receptor and its downstream signalling), as reviewed in [34]. More diverse sub-classification has been reported; for instance Lehmann et al. [35] described six distinct groups exhibiting significantly different signatures for genes controlling proliferation, DNA damage response genes, androgen receptor signalling, and epithelial-mesenchymal transition, with variable responses to different therapies. It is noteworthy that, as gene expression profiling-derived subtypes have arisen from bulk tumour assays using clustering analysis, these represent the average gene expression values for the tumour cells under investigation. Although individual tumours likely display a multitude of features, whether due to intratumoral heterogeneity or to a mixed histotype of the component cells [36], clinical evidence indicates that this does not preclude precise microarray- 
based predictions of tumour behaviour or clinical outcome in BC patients [37].

\section{BC and Tumour Microenvironment (TME)}

The TME is the cellular environment within which the tumour exists including the surrounding blood vessels, either pre-existing or newly formed, immune cells including lymphocytes, macrophages, and other inflammatory cells, fibroblasts, and the ECM. Cumulative evidence has demonstrated that disease outcome is dependent on the intrinsic features of tumour cells, but the TME also actively contributes (94-100 cases). Varying combinations of the different cell types as well as cross-talk cues between them and the tumour cells further modulates the tumour and its surrounding TME, adding more complexity to the heterogeneity and, subsequently, the disease outcome. These tumour-TME interactions are proposed to contribute to early tumorigenesis as well as disease progression [38] and response to therapies [39]. It is wellacknowledged that cancer-associated fibroblasts, immune cells, and vascular/lymphatic endothelial cells (as well as ECM) are known to vary in number and distribution across different tumours and within the same tumour. For instance, increased numbers of tumourassociated macrophages (a highly heterogeneous cellular infiltrate) are associated with a poor prognosis [40], reflecting their ability to enhance BC cell growth [41], angiogenesis [42], and invasion [43]. Angiogenesis, as quantified by microvessel density, is a crucial requisite for tumour growth and is generally accepted as a $\mathrm{BC}$ prognostic factor [32, 33]. However, microvessel density has been reported to have compartment variability of vessel density within the tumour, highlighting the propensity of an invasive front to link to an active process of angiogenesis [44]. In their recent study, Natrajan et al. [45] reported that high microenvironmental diversity was strikingly associated with poor prognosis that could not be explained by tumour size, genomics, or any other data type. This was achieved through their developed quantitative measure of microenvironmental heterogeneity along a 3-spatial-dimensions ecosystem diversity index (EDI) using fully automated histology image analysis. Interestingly, in their study, the prognostic value of EDI was superior to the known prognostic factors, and was further enhanced by the addition of TP53 mutation status, which further supports the integrative cross-talk between the tumour and TME elements. Accordingly, the wide range of different TME components and responses further enhances the heterogeneity of $\mathrm{BC}$, and is a fundamental determinant for the disease course and subsequently the response to different therapeutic regimens.

At the morphological level, there are different types of stroma associated with BC. Some tumours are associated with a florid desmoplastic reaction such as basal-like carcinoma, while other tumours, such as lobular carcinoma, do not initiate a stromal response. Even the desmoplastic stroma associated with BC is different across various tumour types and grades. Low-grade ductal, tubular, and adeno-squamous carcinoma are associated with a desmoplastic, fibrous stromal reaction, which is different from that seen in high-grade tumours such as high-grade ductal/no special type (NST) and basal-like BC. This likely represents different mechanisms of tumour stromal interaction with high-grade, rapidly proliferating tumours associated with hypoxia and tumour necrosis, while lowgrade tumours are slow-growing with no significant hypoxia effect. However, to render the phenomenon of BC heterogeneity even more complex, medullary carcinomas (that are typically high-grade tumours), unlike other high-grade ductal BC, are seldom associated with a stromal desmoplastic response but rather with prominent lymphoid infiltrates.

\section{Intratumoral Heterogeneity and DCIS}

Multiple authorities have reported on the existence of intratumoral heterogeneity in DCIS, the well-known non-obligate precursor of invasive BC [46-48]. It has been proposed that a clonal-selection model of progression similar to that reported in invasive disease takes place in DCIS, and may be responsible for its progression into an overtly invasive tumour despite the conservation of the genomic patterns. For instance, Hernandez et al. [49], using array comparative genomic hybridisation (CGH), FISH, and Sequenom analysis on a subset of DCIS and adjacent invasive cancer showed that both DCIS and the invasive component had strikingly similar genomic profiles; these results were confirmed by other authors [50, 51]. On the other hand, by detecting the patterns of gains and losses using FISH analysis in single cells microdissected from matched DCIS and invasive BC samples, a high level of intratumoral genetic heterogeneity was observed in the two components [52]. Accordingly, although DCIS exhibits genomic patterns that are similar to its matched invasive counterpart, they are non-identical. In addition to the founder genetic aberrations shared by both components, DCIS is proposed to contain a mo-
Aleskandarany/Vandenberghe/Marchiò/ Ellis/Sapino/Rakha 
saic of tumour cells attaining additional mutations, and a progression from in situ to invasive disease occurs in a Darwinian-like clonal selection $[49,53]$. It is noteworthy, however, that there are currently no single morphological or genetic criteria that could be reliably used to predict that a recently diagnosed DCIS case, by, e.g., core-needle biopsy, would progress to invasive disease. Therefore, using a matched case of DCIS and its invasive counterpart is recommended, in order to depict the genetic differences, especially the additional mutational events which may have governed the progression to invasive disease. Moreover, tissue-based assessment of genomic imbalances (like gains and/or losses) appears to be significantly important to map the genetic lesion to the morphology of both the DICS and the invasive components. Importantly, the efficient utilisation of the rapidly advancing, massively parallel sequencing technologies, using dissected tissues and single tumour cells, is important to decipher the molecular pathways and genomic alterations responsible for the evolution of invasive disease from its preinvasive ancestor.

\section{Temporal Heterogeneity and Phenotype Conversion}

It is well recognised that recurrences of $\mathrm{BC}$ at regional or distant metastatic sites may display contrasting characteristics to those found in the primary tumour, manifesting as additional genomic changes [54-56]. For instance, Ellsworth et al. [57] reported genomic discordance between regional metastases and their matched primaries, which was associated with a less favourable outcome. Discordance rates ranging from 13 to $54 \%$ have been reported for ER status and from 0 to 32\% for HER2 status [58]. Moreover, Kurbasic et al. [59] showed that the expression of a large number of glycosylated proteins important in cellular pathways, such as cell adhesion, migration pathways, and immune response, changed between primary tumours and matched lymph node metastases and distant metastases. Although these discrepancies could be attributed to technical issues pertaining to sample preparation/testing, intratumoral heterogeneity is a strong rationale to explain them. The current models of tumour heterogeneity propose that tumour metastases arise from subclones of cells spatially distributed within the primary tumour, which are not necessarily representative of the entire tumour genetic profile. The same phenomenon is observed following neoadjuvant therapy, when the remaining tumour is compared to the primary tumour or pre-treatment core biopsies. This immuno-

Breast Cancer Heterogeneity phenotype drift poses important clinical questions, especially when the phenotype converts from positive to negative (the most frequent scenario), at least for the hormone receptors [60]. Some studies report that receptor conversion by immunohistochemistry (IHC) in nonbone distant $\mathrm{BC}$ metastases is relatively uncommon for ER and HER2, and more frequent for PR, especially in brain, liver, and gastro-intestinal metastases [61]. It is therefore recommended that the management protocol in these cases should be amended based on the status of the emerging metastatic deposits.

\section{Heterogeneity and Tumour Sampling}

There is compelling evidence indicating the usefulness of TMA, the tissue platform commonly used in translational biomarker discovery/validation studies, in a largescale population-based study assessing molecular markers, with a high agreement rate between TMA and fullface sections (FFS) [62]. Using TMA, several groups including ours have reported extensively on the molecular profiling of $\mathrm{BC}$ as well as assessing the prognostic/ predictive value of a variety of biomarkers $[63,64]$. However, it is important to recognise the limitations of this technique, particularly the spatial intratumoral heterogeneity, to avoid misinterpretation of the results. In previous studies, we assessed the expression of different markers including ER, PR (120 cases [63]), the proliferation marker Ki67 (200 cases) [65], and the hypoxia-related marker carbonic anhydrase IX (CAIX) (100 cases [66]), on both TMA and FFS. We noticed that TMA is more likely to produce discrepant results than FFS. Figure 1 shows Ki67 staining of the same case using FFS and TMA. In all the studied markers, the majority of discordant cases were false negative on TMA rather than false positive $[63,67,68]$. It is worth mentioning that, although these discrepancies between TMA and FFS did not significantly alter the overall association of biomarkers like ER and PR with the other prognostic variables, different associations were noted in the case of markers related to cell proliferation and tissue hypoxia, e.g., Ki67 and CAIX, which are more heterogeneous phenomena. Therefore, proper testing of the expression pattern of candidate biomarkers on FFS is highly recommended prior to TMA application. In a case of substantial heterogeneity, FFS would be the recommended platform or at least multiple cores are recommended to minimise the impact of tumour heterogeneity on tissue sampling [69]. Acquiring multiple cores from multiple histologically distinct tumour zones is po- 


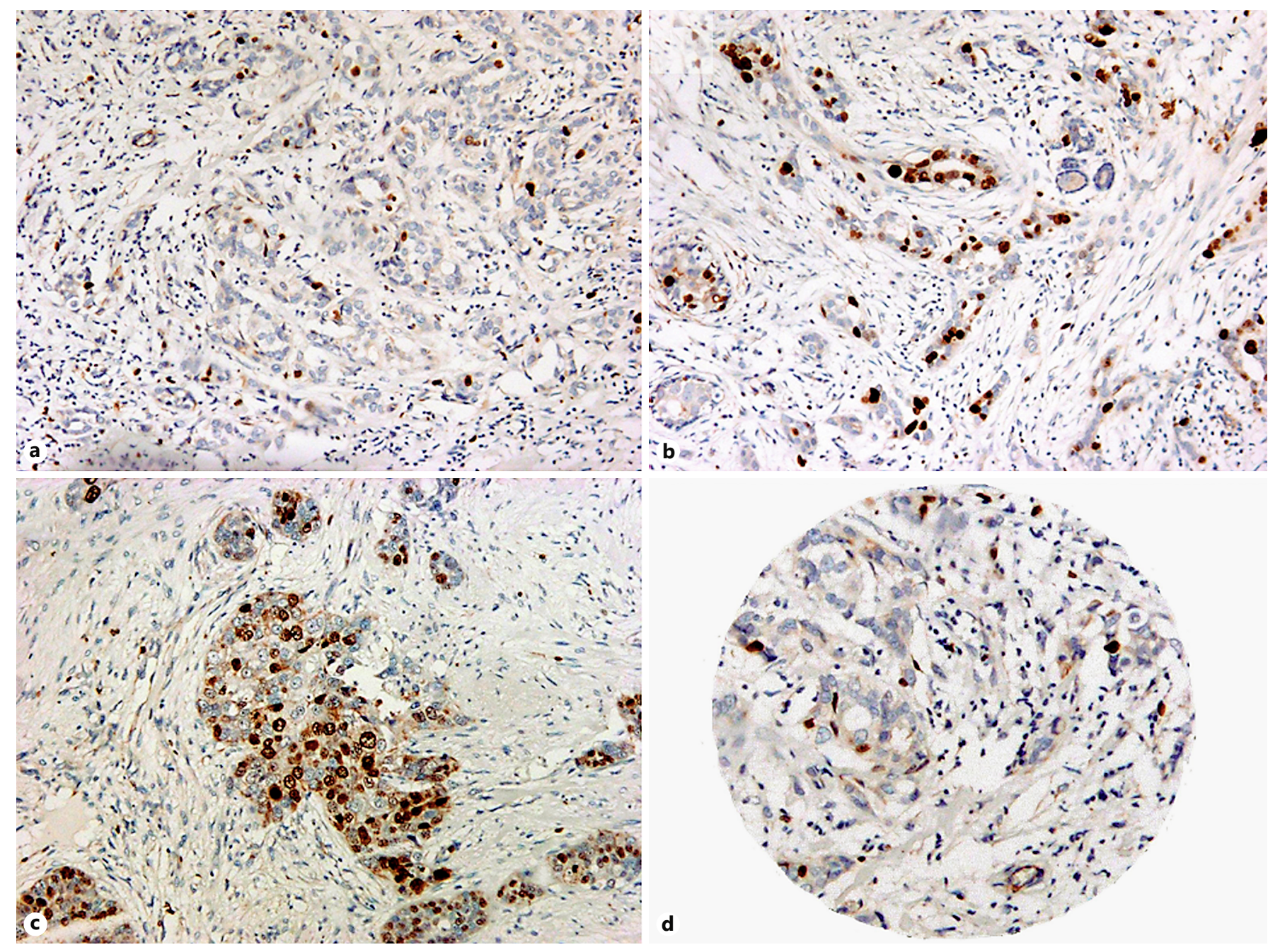

Fig. 1. Immunohistochemical expression of Ki67 in invasive breast cancer. a-c Ki67 expression in different fields from the same case. $\mathbf{d}$ Expression of Ki67 on a tissue microarray core biopsy from the same case.

tentially more effective than increasing the diameter of a single TMA core [70].

From a practical standpoint, it is also important to note that heterogeneity can affect the representation of the whole tumour when it is assessed from the preoperative core biopsies. Not only morphological variables, such as tumour type, grade, and lymphovascular invasion, but also molecular profiles and stroma-related markers such as tumour-infiltrating lymphocytes may be different when compared to surgical excision specimens [71]. Regarding the assessment of biomarkers, there is evidence to indicate the reliability of core biopsies for the assessment of ER and HER2, and, to a lesser extent, PR [71, 72]. It is clear that the representation of core biopsies similar to TMA cores is related to the pattern of biomarker expression, with the homogeneously expressed biomarkers being the most reliable and the least reliability occurring in the heterogeneously expressed biomarkers. The reliability of using core biopsies or TMA for the assessment of ER status to determine the use of hormone therapy is excellent, as ER is typically homogeneous in expression and the cut-off for positivity is very low (1\%) [73]. However, in very exceptional situations, an ER-positive clone constituting a minority of $\mathrm{BC}$ cells could be detected (Fig. 2). On the other hand, heterogeneously expressed markers such as E-cadherin, PR, Ki67, and hypoxia-related marker [74, 75] could be less reliably assessed using TMA or core biopsies. 


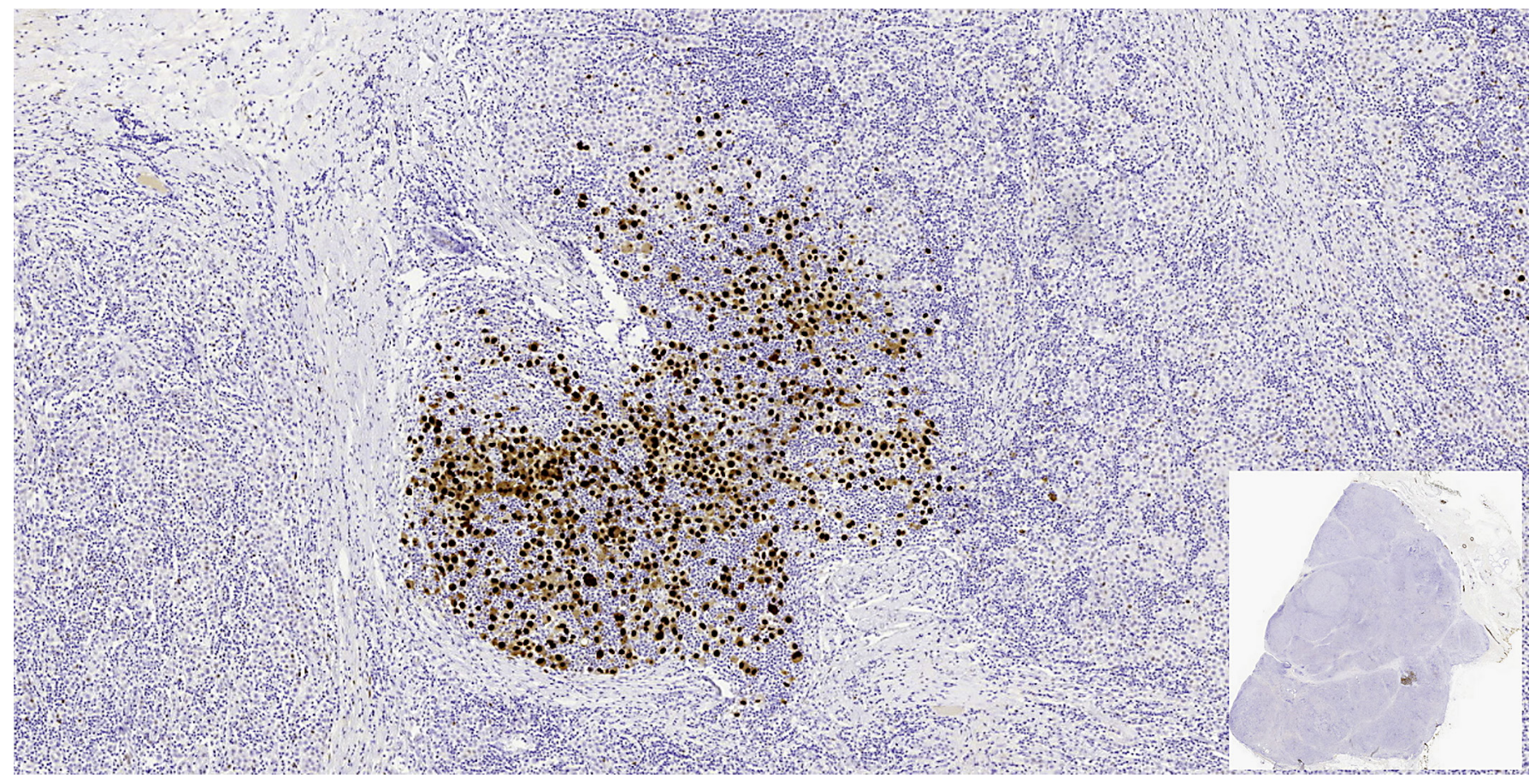

Fig. 2. Immunohistochemical expression of the oestrogen receptor in a case of invasive BC, showing an ER-positive clone forming a minority of cells within the vast majority of ER-negative BC cells. Inset $\times 10$. BC, breast cancer; ER, oestrogen receptor.

\section{Heterogeneity and Circulating Tumour Cells}

Novel tumour-sampling techniques such as analysis of circulating tumour cells (CTCs) or circulating tumour DNA, may allow a more comprehensive analysis of the clonal composition of $\mathrm{BC}$ provided that cancer cells are shed into the circulation proportional to their clonal frequency in the primary. CTC can also reflect the existence of temporal heterogeneity [76]. In general, CTCs show a considerable intrapatient heterogeneity, which may reflect the temporal evolution of tumour cell subclones over time [77] or an expansion of treatment-resistant cells that escaped systemic therapy effects [76]. It has been suggested that CTCs might provide a more biological and clinical description of heterogeneity of the entire tumour burden within a particular patient versus bulk biopsies from the primary tumour or individual metastatic lesions. Reliable and accurate measurement and molecular characterisation of CTCs utilising a variety of detection techniques for multiple protein biomarkers including ER, HER2 expression, Ki67, BCL2, and HER2 amplification status have been reported [78-81]. Although CTCs are regarded as a powerful marker for the prediction of early metastatic disease [82-84], currently, sound evidence of the utility of assessed biomarkers utilising CTC to predict clinical response or patients' outcome is still lacking. For instance, it has been reported that HER2 positivity in CTCs was not predictive to the response to lapatinib [85]. Moreover, the occurrence of genetic switches within some tumour cells sub-clones, e.g., epithelial-mesenchymal transition, could potentially explain why the molecular features of CTCs may not be fully representative of the heterogeneous genetic/molecular portrait and its subsequent impact on disease outcome.

\section{Heterogeneity and the Efficacy of Targeted Therapy}

Significant intratumoral genetic heterogeneity is likely to limit the efficacy of targeted therapies even prior to the acquisition of drug resistance. For instance, the Darwinian-like selection [86] of pre-existing drug-resistant cell clones could contribute to the initial therapeutic resistance, and could also explain the emergence of such clones, due to genomic instability over the course of time in the progression of a tumour [87]. Resistance to 
poly(ADP) ribose polymerase inhibitors (PARP inhibitors) and platinum-based chemotherapeutic regimens in $\mathrm{BC}$ patients harbouring a $B R C A 1$ or $B R C A 2$ germline mutation was reported to be mediated in a proportion of cases through the acquisition of revertant mutations or intragenic deletions that restore the open reading frame of BRCA1 or BRCA2 genes [88]. However, based on currently available data, these secondary somatic genetic events affecting $B R C A 1$ or $B R C A 2$ might have preceded the therapeutic intervention [89]. Whether these therapyresistance-causing mutations occurred prior to or during the course of therapy, they reflect significant intratumoral genetic heterogeneity which subsequently resulted in targeted therapy failure.

Elimination of sensitive tumour cell clones and/or their adaptation in response to therapeutic agents will not only explain the acquired resistance but also fuel intratumoral heterogeneity [90]. Therefore, studies on the genetic landscape of cancer and biomarker discovery/validation as well as therapy planning for individual patients may be compromised by using a single tumour biopsy specimen, with the potential to overlook or at least underestimate the burden of heterogeneous tumours [91].

Heterogeneity may represent a major issue in the neoadjuvant setting where core biopsies of large lesions are studied to plan pre-surgery therapy. In this scenario, multiple biopsies are suggested to avoid improper sampling and to properly assess morphological and, more importantly, immunophenotypical features [92]. Among the 4 routinely tested predictive markers, ER is reported to be the least heterogeneous, being expressed either at high or very low levels [93]; the current cut-off of ER immunohistochemical positivity is set at a low level (1\%), which makes core biopsy a sensitive and specific method for its assessment. Although PR expression can be heterogeneous [94], it is unlikely to affect treatment decisions since it is ER-dependent and the ER-negative PR-positive phenotype is rare. Ki67 is another marker of clinical relevance that suffers from intratumoral heterogeneity [95].

Recently, we evaluated the expression of Ki67 in multiple areas from a subset of primary invasive BC and in the corresponding lymph node metastasis [96], and found a high degree of variation in the expression across the areas on the slide from the same tumour block as well as between slides from different tumour blocks. Interestingly, the highest scores of Ki67 in primary tumours coincided with those in the nodal metastatic deposits (Fig. 3). Our findings demonstrated the spatial heterogeneity, shown in the intra- and interslide variable Ki67 expression, as well as temporal heterogeneity, shown in nodal deposits that could represent the clonal expansion of the highly proliferative primary tumour subclones.

By definition, HER2 positivity is heterogeneous, since a $10 \%$ cut-off is considered to assign samples to distinct score categories using IHC [97]; scores of 0 and $3+$ are more homogeneous in staining and $2+$ carcinomas show higher variability [98]. HER2 heterogeneity is known to feature either 2 clearly distinct tumour clones or scattered HER2-positive cells within a substantially negative tumour cell population [99]. Recently, the mutational landscape of HER 2 heterogeneous carcinomas with 2 separate clones was analysed, and distinct driver genetic alterations in the different components were demonstrated, suggesting that HER2-negative clones are likely driven by genetic alterations not present in the HER2-positive clones, including the BRF2 and DSN1 amplification and the HER2 I767M somatic mutations [98]. Accordingly, biological marker heterogeneity suggests that multiple cores should be obtained for using TMA procedure in the routine and research settings to represent all the possible variability of expression [100].

\section{Methods to Quantify Spatial Intratumoral Heterogeneity}

In studies evaluating intratumoral heterogeneity, investigators typically record the relative abundance of different cell populations within a tumour. Based on the distribution of different cell populations, a variety of approaches can be employed to measure the extent of heterogeneity. The choice of approach to measure intratumoral heterogeneity can be somewhat confusing, but is crucial as it can directly impact study results [99]. The choice of the appropriate methodology depends on 3 factors: (i) the importance given to rare cell populations versus abundant cell populations, (ii) whether similarities exist across different cell populations, and (iii) the spatial scale(s) at which heterogeneity is quantified. Ecologists have studied diversity within biological systems for decades, resulting in the development of a number of diversity measures; these measures were adapted to quantify genetic [21], molecular [99, 101, 102], and microenvironmental [45] heterogeneity within breast tumours. The most cited measures are the Shannon entropy [103], the Simpson index [104], and the Rao quadratic entropy [105]. The Shannon entropy and the Simpson index have been shown to belong to the same family of diversity measures, differing only in their sensitivity to rare populations [106]. Measures that are more sensitive to rare pop- 




Fig. 3. Immunohistochemical heterogeneity of Ki67 in breast cancer. a, b Variable expression of Ki67 in different tumour blocks from the same case. c, d Another example of variable expression of Ki67 in full-face sections from different primary tumour sections. Inset Ki67 expression in an axillary node from the same case.

ulation of cells, such as the Shannon entropy, are particularly relevant in the context of evaluating the impact of intratumoral heterogeneity on the efficacy of targeted therapies because treatment resistance is thought to originate from small colonies of resistant cells. In contrast, the confounding effect of intratumoral heterogeneity on diagnosis is thought to rather be caused by the presence of large cell populations that are not adequately reflected by tumour sampling [99].

Another important factor to consider when quantifying heterogeneity is whether similarities exist between different cell populations within the total tumour cell population. For instance, a tumour whose HER2
IHC score is $50 \%$ negative (0) and 50\% $3+$ is arguably more heterogeneous than a tumour whose HER2 IHC score is $50 \% 2+$ and $50 \% 3+$ because $2+$ and $3+$ tumour cells are more similar than 0 and $3+$ tumour cells. The Shannon entropy and the Simpson index, along with affiliated measures, do not properly account for potential similarities between cell types, and the examples cited above would be considered by those measures as equally heterogeneous. Provided that similarities between cell types can be explicitly specified, a more relevant heterogeneity measure can be obtained by using the Rao quadratic entropy which would appropriately reflect the similarities. 
Finally, intratumoral heterogeneity can be quantified at various spatial scales. Potts et al. [99] observed that global HER2 expression heterogeneity at the tumour level affects agreement between pathologists but that local heterogeneity between cells that are in close proximity does not. This suggests that local heterogeneity quantification may not be relevant when studying the impact of intratumoral heterogeneity on diagnosis. Interestingly, they also found that global HER2 expression heterogeneity is not correlated with local HER2 heterogeneity, indicating that global heterogeneity and local heterogeneity arise from distinct mechanisms. This suggests that, at least in the case of HER2 expression, local heterogeneity may be an aspect of tumour biology, with distinct implications for the efficacy of targeted therapies, but this remains to be demonstrated.

Taken together, the whole panorama of morphological, immunophenotypical, and molecular features described here for accurately assessing the extent and clinical impact of heterogeneity in BC should be taken into account when high-throughput molecular studies are performed.
From a practical standpoint, whether in the standard routine practice or research setting, we suggest that:

- histomorphological heterogeneity is not a rare event in $\mathrm{BC}$ and it may influence patient prognosis and response to treatment;

- examination of $>1$ section of tumour by means of $\mathrm{H} \& \mathrm{E}$ staining is mandatory prior to assessing biomarker expression known or proved to be heterogeneous;

- IHC assessment of prognostic/predictive markers should be performed on the more heterogeneous section at the H\&E examination;

- when defining neoadjuvant chemotherapy protocols, $>1$ core biopsy should be taken to adequately assess predictive marker status for guiding therapy;

- PR and Ki67 are heterogeneously expressed, and should be assessed on whole sections to avoid misleading results which could arise from suboptimal sampling (e.g., in TMA);

- molecular studies that plan to report on heterogeneity should attentively consider morphological and immunophenotypical heterogeneity in the experimental design and the interpretation of results.

\section{References}

1 Marusyk A, Almendro V, Polyak K: Intratumour heterogeneity: a looking glass for cancer? Nat Rev Cancer 2012;12:323-334.

$>2$ Navin N, et al: Inferring tumor progression from genomic heterogeneity. Genome Res 2010;20:68-80.

$>3$ Ding L, et al: Genome remodelling in a basallike breast cancer metastasis and xenograft. Nature 2010;464:999-1005.

-4 Ellis IO, et al: Pathological prognostic factors in breast cancer. II. Histological type. Relationship with survival in a large study with long-term follow-up. Histopathology 1992; 20:479-489.

5 Lakhani SR, et al (eds): WHO Classification of Tumours of the Breast, ed 4. Lyon, IARC, 2012.

-6 Elston CW, Ellis IO: Pathological prognostic factors in breast cancer. I. The value of histological grade in breast cancer: experience from a large study with long-term follow-up. Histopathology 1991;19:403-410.

7 Goldhirsch A, et al: Strategies for subtypes dealing with the diversity of breast cancer: highlights of the St. Gallen International Expert Consensus on the Primary Therapy of Early Breast Cancer 2011. Ann Oncol 2011; 22:1736-1747.

$>8$ Rakha EA, et al: Tubular carcinoma of the breast: further evidence to support its excellent prognosis. J Clin Oncol 2010;28:99-104.

$\checkmark 9$ Sinn H-P, Kreipe H: A brief overview of the WHO Classification of Breast Tumors, 4th edition, focusing on issues and updates from $>19$ Burrell RA, Swanton C: Tumour heterogenethe 3rd edition. Breast Care 2013;8:149-154.

10 Geyer FC, et al: Molecular analysis reveals a genetic basis for the phenotypic diversity of metaplastic breast carcinomas. J Pathol 2010; 220:562-573.

$\checkmark 11$ Hennessy BT, et al: Characterization of a naturally occurring breast cancer subset enriched in epithelial-to-mesenchymal transition and stem cell characteristics. Cancer Res 2009;69: 4116-4124.

12 van Deurzen CH, et al: Metaplastic breast carcinoma: tumour histogenesis or dedifferentiation? J Pathol 2011;224:434-437.

13 Wang X, et al: Metaplastic carcinoma of the breast: p53 analysis identified the same point mutation in the three histologic components. Mod Pathol 2001;14:1183-1186.

14 Molyneux G, et al: BRCA1 basal-like breast cancers originate from luminal epithelial progenitors and not from basal stem cells. Cell Stem Cell 2010;7:403-417.

$>15$ Verigos J, Magklara A: Revealing the complexity of breast cancer by next generation sequencing. Cancers (Basel) 2015;7:2183-2200.

16 Meacham CE, Morrison SJ: Tumor heterogeneity and cancer cell plasticity. Nature 2013; 501:328-337.

17 Greaves M, Maley CC: Clonal evolution in cancer. Nature 2012;481:306-313.

18 Marusyk A, Polyak K: Tumor heterogeneity: causes and consequences. Biochim Biophys Acta 2010;1805:105. ity and the evolution of polyclonal drug resistance. Mol Oncol 2014;8:1095-1111.

20 Schiavon G, et al: Analysis of ESR1 mutation in circulating tumor DNA demonstrates evolution during therapy for metastatic breast cancer. Sci Transl Med 2015;7:313ra182.

21 Park SY, et al: Cellular and genetic diversity in the progression of in situ human breast carcinomas to an invasive phenotype. J Clin Invest 2010;120:636-644.

22 Perou CM, et al: Molecular portraits of human breast tumours. Nature 2000;406:747752 .

23 Sørlie T, et al: Gene expression patterns of breast carcinomas distinguish tumor subclasses with clinical implications. Proc Natl Acad Sci USA 2001;98:10869-10874.

24 Sørlie T, et al: Repeated observation of breast tumor subtypes in independent gene expression data sets. Proc Natl Acad Sci USA 2003; 100:8418-8423.

25 Reis-Filho JS, Pusztai L: Gene expression profiling in breast cancer: classification, prognostication, and prediction. Lancet 2011;378: 1812-1823.

26 Prat A, et al: Clinical implications of the intrinsic molecular subtypes of breast cancer. Breast 2015;24(suppl 2):S26-S35.

27 Curtis $C$, et al: The genomic and transcriptomic architecture of 2,000 breast tumours reveals novel subgroups. Nature 2012;486:346352 . 
28 Blows FM, et al: Subtyping of breast cancer by immunohistochemistry to investigate a relationship between subtype and short and long term survival: a collaborative analysis of data for 10,159 cases from 12 studies. PLoS Med 2010;7:e1000279.

29 Nielsen TO, et al: Immunohistochemical and clinical characterization of the basal-like subtype of invasive breast carcinoma. Clin Cancer Res 2004;10:5367.

30 Rakha EA, et al: Triple-negative breast cancer: distinguishing between basal and nonbasal subtypes. Clin Cancer Res 2009;15:23022310.

31 Habashy HO, et al: A review of the biological and clinical characteristics of luminal-like oestrogen receptor-positive breast cancer. Histopathology 2012;60:854-863.

32 Uzzan B, et al: Microvessel density as a prognostic factor in women with breast cancer: a systematic review of the literature and metaanalysis. Cancer Res 2004;64:2941-2955.

- 33 Mohammed RA, et al: Lymphatic and angiogenic characteristics in breast cancer: morphometric analysis and prognostic implications. Breast Cancer Res Treat 2009;113:261273.

34 Badve S, et al: Basal-like and triple-negative breast cancers: a critical review with an emphasis on the implications for pathologists and oncologists. Mod Pathol 2011;24:157167.

-35 Lehmann BD, et al: Identification of human triple-negative breast cancer subtypes and preclinical models for selection of targeted therapies. J Clin Invest 2011;121:2750-2767.

-36 Navin N, et al: Tumour evolution inferred by single-cell sequencing. Nature 2011;472:9094.

-37 Barry WT, et al: Intratumor heterogeneity and precision of microarray-based predictors of breast cancer biology and clinical outcome. J Clin Oncol 2010;28:2198-2206.

- 38 Schedin P, Elias A: Multistep tumorigenesis and the microenvironment. Breast Cancer Res 2004;6:1-9.

- 39 Wu T, Dai Y: Tumor microenvironment and therapeutic response. Cancer Lett 2017;387: 61-68.

40 Hao N-B, et al: Macrophages in tumor microenvironments and the progression of tumors. Clin Dev Immunol 2012;2012:11.

41 Tsutsui S, et al: Macrophage infiltration and its prognostic implications in breast cancer: the relationship with VEGF expression and microvessel density. Oncol Rep 2005; 14:425431.

42 Murdoch C, et al: The role of myeloid cells in the promotion of tumour angiogenesis. Nat Rev Cancer 2008;8:618-631.

-43 Siveen KS, Kuttan G: Role of macrophages in tumour progression. Immunol Lett 2009;123: 97-102.

44 Haisan A, et al: Digital microscopy assessment of angiogenesis in different breast cancer compartments. Biomed Res Int 2013; 2013:286902.
45 Natrajan R, et al: Microenvironmental heterogeneity parallels breast cancer progression: a histology-genomic integration analysis. PLoS Med 2016;13:e1001961.

46 Aubele M, et al: Extensive ductal carcinoma in situ with small foci of invasive ductal carcinoma: evidence of genetic resemblance by CGH. Int J Cancer 2000;85:82-86.

$47 \mathrm{Hu} \mathrm{M}$, et al: Regulation of in situ to invasive breast carcinoma transition. Cancer Cell 2008;13:394-406.

48 Clark SE, et al: Molecular subtyping of DCIS: heterogeneity of breast cancer reflected in pre-invasive disease. Br J Cancer 2011;104: 120-127.

49 Hernandez L, et al: Genomic and mutational profiling of ductal carcinomas in situ and matched adjacent invasive breast cancers reveals intra-tumour genetic heterogeneity and clonal selection. J Pathol 2012;227:42-52.

50 Krøigård $\mathrm{AB}$, et al: Clonal expansion and linear genome evolution through breast cancer progression from pre-invasive stages to asynchronous metastasis. Oncotarget 2015;6: 5634-5649.

51 Kim SY, et al: Genomic differences between pure ductal carcinoma in situ and synchronous ductal carcinoma in situ with invasive breast cancer. Oncotarget 2015;6:7597-7607.

52 Heselmeyer-Haddad K, et al: Single-cell genetic analysis of ductal carcinoma in situ and invasive breast cancer reveals enormous tumor heterogeneity yet conserved genomic imbalances and gain of MYC during progression. Am J Pathol 2012;181:1807-1822.

53 Sakr RA, et al: PI3K pathway activation in high-grade ductal carcinoma in situ - implications for progression to invasive breast carcinoma. Clin Cancer Res 2014;20:2326-2337.

54 Manso L, et al: Analysis of paired primarymetastatic hormone-receptor positive breast tumors (HRPBC) uncovers potential novel drivers of hormonal resistance. PLoS One 2016;11:e0155840.

55 Simmons C, et al: Does confirmatory tumor biopsy alter the management of breast cancer patients with distant metastases? Ann Oncol 2009;20:1499-1504.

56 Macfarlane R, et al: Molecular alterations between the primary breast cancer and the subsequent locoregional/metastatic tumor. Oncologist 2012;17:172-178.

57 Ellsworth RE, et al: Molecular heterogeneity in primary breast carcinomas and axillary lymph node metastases assessed by genomic fingerprinting analysis. Cancer Growth $\mathrm{Me}$ tastasis $2015 ; 8: 15-24$.

58 Arslan C, et al: Variation in hormone receptor and HER-2 status between primary and metastatic breast cancer: review of the literature. Expert Opin Ther Targets 2011;15:21-30.

59 Kurbasic E, et al: Changes in glycoprotein expression between primary breast tumour and synchronous lymph node metastases or asynchronous distant metastases. Clin Proteomics 2015;12:13.
60 Cummings MC, et al: Metastatic progression of breast cancer: insights from 50 years of autopsies. J Pathol 2014;232:23-31.

61 Hoefnagel LD, et al: Receptor conversion in distant breast cancer metastases. Breast Cancer Res 2010;12:R75.

62 Zhang D, et al: Reliability of tissue microarrays in detecting protein expression and gene amplification in breast cancer. Mod Pathol 2003;16:79-85.

63 Rakha EA, et al: Biologic and clinical characteristics of breast cancer with single hormone receptor positive phenotype. J Clin Oncol 2007;25:4772-4778.

64 Ruiz C, et al: Tissue microarrays for comparing molecular features with proliferation activity in breast cancer. Int J Cancer 2006;118: 2190-2194.

65 Aleskandarany M, et al: MIB1 tumour growth fraction assessment in lymph node negative breast cancer. J Pathol 2008;216(S1):S9.

66 Lancashire L, et al: Neural networks identify gene profiles that predict clinical outcome with high accuracy in breast cancer: CA IX is identified as the principal prognostic indicator. J Pathol 2008;216(S1):S49.

67 Aleskandarany MA, et al: Prognostic value of proliferation assay in the luminal, HER2-positive, and triple-negative biologic classes of breast cancer. Breast Cancer Res 2012;14:R3.

68 Lancashire LJ, et al: A validated gene expression profile for detecting clinical outcome in breast cancer using artificial neural networks. Breast Cancer Res Treat 2010;120:83-93.

69 Voduc D, Kenney C, Nielsen TO: Tissue microarrays in clinical oncology. Semin Radiat Oncol 2008;18:89-97.

70 Anagnostou VK, et al: Quantitative evaluation of protein expression as a function of tissue microarray core diameter: is a large (1.5 $\mathrm{mm})$ core better than a small $(0.6 \mathrm{~mm})$ core? Arch Pathol Lab Med 2010;134:613-619.

71 Rakha EA, Ellis IO: An overview of assessment of prognostic and predictive factors in breast cancer needle core biopsy specimens. J Clin Pathol 2007;60:1300-1306.

72 Rakha EA, et al: Updated UK recommendations for HER2 assessment in breast cancer. J Clin Pathol 2015;68:93-99.

73 Hammond ME, et al: American Society of Clinical Oncology/College of American Pathologists guideline recommendations for immunohistochemical testing of estrogen and progesterone receptors in breast cancer. J Clin Oncol 2010;28:2784-2795.

74 Rakha EA, et al: E-cadherin expression in invasive non-lobular carcinoma of the breast and its prognostic significance. Histopathology 2005;46:685-693.

75 Rakha EA, Ellis IO: Does estrogen receptor-negative/progesterone receptor-positive breast carcinoma exist? In reply. J Clin Oncol 2008;26:335-336.

76 Babayan A, et al: Heterogeneity of estrogen receptor expression in circulating tumor cells from metastatic breast cancer patients. PLoS One 2013;8:e75038. 
77 Hayes DF, Paoletti C: Circulating tumour cells: insights into tumour heterogeneity. J Intern Med 2013;274:137-143.

-78 Fehm T, et al: ERalpha-status of disseminated tumour cells in bone marrow of primary breast cancer patients. Breast Cancer Res 2008; 10:R76.

79 Fehm T, et al: Determination of HER2 status using both serum HER2 levels and circulating tumor cells in patients with recurrent breast cancer whose primary tumor was HER2 negative or of unknown HER2 status. Breast Cancer Res 2007;9:R74.

80 Paoletti C, et al: Development of circulating tumor cell-endocrine therapy index in patients with hormone receptor-positive breast cancer. Clin Cancer Res 2015;21:2487-2498.

81 Krishnamurthy S, et al: Discordance in HER2 gene amplification in circulating and disseminated tumor cells in patients with operable breast cancer. Cancer Med 2013;2:226-233.

82 Giuliano M, et al: Circulating tumor cells as early predictors of metastatic spread in breast cancer patients with limited metastatic dissemination. Breast Cancer Res 2014;16:1-9.

83 Giuliano M, et al: Circulating tumor cells as prognostic and predictive markers in metastatic breast cancer patients receiving firstline systemic treatment. Breast Cancer Res 2011;13:R67.

-84 Bidard FC, et al: Clinical validity of circulating tumour cells in patients with metastatic breast cancer: a pooled analysis of individual patient data. Lancet Oncol 2014;15:406-414.

-85 Pestrin M, et al: Final results of a multicenter phase II clinical trial evaluating the activity of single-agent lapatinib in patients with HER2negative metastatic breast cancer and HER2positive circulating tumor cells. A proof-ofconcept study. Breast Cancer Res Treat 2012; 134:283-289.
86 Gerlinger M, et al: Intratumor heterogeneity and branched evolution revealed by multiregion sequencing. N Engl J Med 2012;366:883892.

87 Lee AJ, et al: Chromosomal instability confers intrinsic multidrug resistance. 2011;71:18581870.

88 Edwards SL, et al: Resistance to therapy caused by intragenic deletion in BRCA2. Nature 2008;451:1111-1115.

89 Yamamoto KN, et al: Evolution of pre-existing versus acquired resistance to platinum drugs and PARP inhibitors in BRCA-associated cancers. PLoS One 2014;9:e105724.

90 de Gramont A, et al: Pragmatic issues in biomarker evaluation for targeted therapies in cancer. Nat Rev Clin Oncol 2015;12:197-212.

91 Martelotto LG, et al: Breast cancer intra-tumor heterogeneity. Breast Cancer Res 2014; 16:210-210.

92 Marchio C, Sapino A: The pathologic complete response open question in primary therapy. J Natl Cancer Inst Monogr 2011;2011:86-90.

93 Collins LC, Boterov ML, Schnitt SJ: Bimodal frequency distribution of estrogen receptor immunohistochemical staining results in breast cancer: an analysis of 825 cases. Am J Clin Pathol 2005;123:16-20.

94 Torhorst J, et al: Tissue microarrays for rapid linking of molecular changes to clinical endpoints. Am J Pathol 2001;159:2249-2256.

95 Dowsett M, et al: Assessment of Ki67 in breast cancer: recommendations from the International Ki67 in Breast Cancer working group. J Natl Cancer Inst 2011;103:1656-1664.

96 Aleskandarany MA, et al: Impact of intratumoral heterogeneity on the assessment of Ki67 expression in breast cancer. Breast Cancer Res Treat 2016;158:287-295.
97 Wolff AC, et al: Recommendations for human epidermal growth factor receptor 2 testing in breast cancer: American Society of Clinical Oncology/College of American Pathologists clinical practice guideline update. J Clin Oncol 2013;31:3997-4013.

$\$ 98 \mathrm{Ng}$ CK, et al: Intra-tumor genetic heterogeneity and alternative driver genetic alterations in breast cancers with heterogeneous HER2 gene amplification. Genome Biol 2015;16:107.

99 Potts SJ, et al: Evaluating tumor heterogeneity in immunohistochemistry-stained breast cancer tissue. Lab Invest 2012;92:13421357.

100 Sapino A, et al: Routine assessment of prognostic factors in breast cancer using a multicore tissue microarray procedure. Virchows Arch 2006;449:288-296.

101 Faratian D, et al: Heterogeneity mapping of protein expression in tumors using quantitative immunofluorescence. J Vis Exp 2011;56:e3334.

102 Almendro V, et al: Inference of tumor evolution during chemotherapy by computational modeling and in situ analysis of genetic and phenotypic cellular diversity. Cell Rep 2014;6:514-527.

103 Shannon C, Weaver W (eds): The Mathematical Theory of Communication. Urbana, University of Illinois Press, 1949, pp 3-91.

104 Simpson E: Measurement of diversity. Nature 1949;163:688-688.

105 Rao CR: Diversity and dissimilarity coefficients: a unified approach. Theor Popul Biol 1982;21:24-43.

106 Hill MO: Diversity and evenness: a unifying notation and its consequences. Ecology 1973;54:427-432. 